\title{
Low-temperature creation of Frenkel defects via hot electron-hole recombination in highly pure $\mathrm{NaCl}$ single crystals
}

\author{
A. Lushchik, Ch. Lushchik, V. Nagirnyi, E. Shablonin, and E. Vasil'chenko \\ Institute of Physics, University of Tartu, W. Ostwald Str. 1, Tartu 50411, Estonia \\ E-mail: aleksandr.lushchik@ut.ee
}

Received January 25, 2016, published online May 25, 2016

\begin{abstract}
The creation spectrum of stable $F$ centres (being part of $F-H$ pairs of Frenkel defects) by synchrotron radiation of 7-40 eV has been measured for highly pure $\mathrm{NaCl}$ single crystals at $12 \mathrm{~K}$ using a highly sensitive luminescent method. It is shown that the efficiency of $F$ centre creation in a closely packed $\mathrm{NaCl}$ is low at the decay of anion or cation excitons (7.8-8.4 and $33.4 \mathrm{eV}$, respectively) or at the recombination of relaxed conduction electrons and valence holes. Only the recombination of nonrelaxed (hot) electrons with holes provides the energy exceeding threshold value $E_{F D}$, which is sufficient for the creation of Frenkel defects at low temperature.

PACS: 61.82.Ms Insulators;

78.55.Fv Solid alkali halides;

78.60.Hk Cathodoluminescence, ionoluminescence.
\end{abstract}

Keywords: Frenkel defects, point defect creation, photoluminescence, alkali halides.

\section{Introduction}

To meet the needs of several applications it is necessary to increase the radiation resistance of inorganic wide-gap materials (WGMs, $E_{g}>5 \mathrm{eV}$ ). The coloration of $\mathrm{NaCl}$ model crystals under $\mathrm{x}$ rays was investigated already by Röntgen and Ioffe more than hundred years ago, but a systematic study of radiation effects/defects was started in the middle of the 20 century. The energy absorbed by the material during irradiation is only partly spent on the excitation of emissions sometimes useful for various applications, while a significant part of the gained energy is transformed via nonradiative transitions into heat (package of phonons) or structural defects. The latter includes both transformation of the existing pre-irradiation defects and the creation of novel short-lived and long-lived (stable) lattice defects.

It is commonly accepted that two groups of mechanisms are responsible for the creation of Frenkel defects (FDs, interstitial-vacancy pairs) in WGMs (see [1-3] and references therein). A knock-out (impact) mechanism is connected with elastic collisions of high-energy incident particles with the crystal nuclei, providing the displacement of atoms from their regular lattice sites into interstices with the formation of pairs of FDs. This universal for solids mechanism is very rapid $\left(10^{-15} \mathrm{~s}\right)$ and plays the dominant role in radiation damage of metals and alloys. However, nonimpact mechanisms connected with the excitation and ionization of an electron system, i.e., the formation of radiation-induced electronic excitations (EEs), are rather efficient in many WGMs [1-3].

The ratio between impact and nonimpact mechanisms depends on many factors, including the value of the threshold energy for the creation of an FD pair $\left(E_{F D}\right)$ with respect to the energy gap. The inequality $E_{F D}<E_{g}$ is valid for many WGMs (incl. the majority of alkali halides) and FDs in these so-called radiation-sensitive materials are efficiently formed at the recombination of totally relaxed conduction electrons $(e)$ and valence holes $(h)$ and even at the decay of self-trapping excitons [1-7]. In many metal oxides considered as radiation-resistant materials, $E_{F D}>E_{g}$, and the above-mentioned nonimpact mechanisms are not realized due to energetic reasons. However, the energy released at the recombination of nonrelaxed (hot) $e$ and $h$ can exceed $E_{F D}$, and FDs in radiation-resistant WGMs are formed via hot $e-h$ recombination, the probability of which significantly increases under conditions of high-dense excitation. Recently, the manifestations of radiation damage via EE-related mechanisms have been detected in a number of metal oxides under irradiation with $\sim \mathrm{GeV}$ swift 
heavy ions that provide extremely high density of EEs within cylindrical ion tracks (see [8-15] and references therein).

It is worth noting that among model alkali halide crystals there exists a closely-packed $\mathrm{NaCl}$ crystal where the inequality $E_{F D}>E_{g}$ is valid below $120 \mathrm{~K}$, and the efficiency of stable FD pair creation (neutral $F-H$ pairs) at low temperatures is low $[2,16,17]$. The excitonic and $e-h$ processes as well as the creation of FDs in pure and doped $\mathrm{NaCl}$ crystals have been studied for a long time [18-32]. Because of the great difference in radii of sodium and chlorine ions, the interstices in the closely packed $\mathrm{NaCl}$ lattice are small that impedes the displacement of anions from regular sites with the formation of interstitials. Furthermore, in contrast to the majority of alkali halides, $H$ centres (a dihalide $\mathrm{X}_{2}^{-}$molecule located at one anion site) in $\mathrm{NaCl}$ are oriented along $<111>$ directions [1,6,24]. The latter circumstance prevents crowdion-type low-temperature migration of $H$ centres from geminate $F$ centres with the formation of pairs of stable (spatially separated) FDs $[1,6]$.

The aim of the present study is to consider experimental manifestations of the photocreation of $F-H$ pairs via hot $e-h$ recombination in a wide region of exciting photon energy $(7-40 \mathrm{eV})$ at low temperature. Namely stable $\left(\tau>10^{3} \mathrm{~s}\right)$ FDs that play a crucial role in WGM degradation during prolonged irradiation are the subject of this study although the number of short-lived defects $\left(\tau \sim 10^{-10}-10^{1} \mathrm{~s}\right)$ is usually significantly higher (see, e.g., [1,2] and references therein).

\section{Experimental}

$\mathrm{NaCl}$ single crystals were grown by the Stockbarger method from highly pure salt after a melt treatment in $\mathrm{Cl}_{2}$ gas flow and a manyfold recrystallization from the melt [24]. The content of divalent metal impurities was less than $0.5 \mathrm{ppm}$, only the amount of $\mathrm{Ca}^{2+}$ was not less than $10 \mathrm{ppm}$. Single $\mathrm{NaCl}$ :Ag crystals containing $60 \mathrm{ppm}$ of $\mathrm{Ag}^{+}$impurity ions and $\mathrm{NaCl}: \mathrm{Br}$ crystals with about $50 \mathrm{ppm}$ of $\mathrm{Br}^{-}$ were studied as well.

The photoluminescence experiments were carried out using synchrotron radiation (SR) facilities in Hamburg (SUPERLUMI station of HASYLAB at DESY) and Lund (the undulator beamline I3 at MAX-III Laboratory). The reflection and excitation spectra were normalized (using the signal from a sodium salicylate coated mesh) to equal quantum intensities of SR falling onto the crystal. The creation spectrum of $F$ centres by SR was measured using a highly sensitive luminescence method elaborated in Tartu and used earlier for the analysis of FD creation in alkali halides and other wide-gap materials (see, e.g., $[4,5,19,24,29,33])$.

The geometry for measuring the creation spectrum, a typical time dependence of the detected luminescence intensity as well as the stimulation spectrum of this luminescence are shown in Fig. 1. The sample was irradiated (time

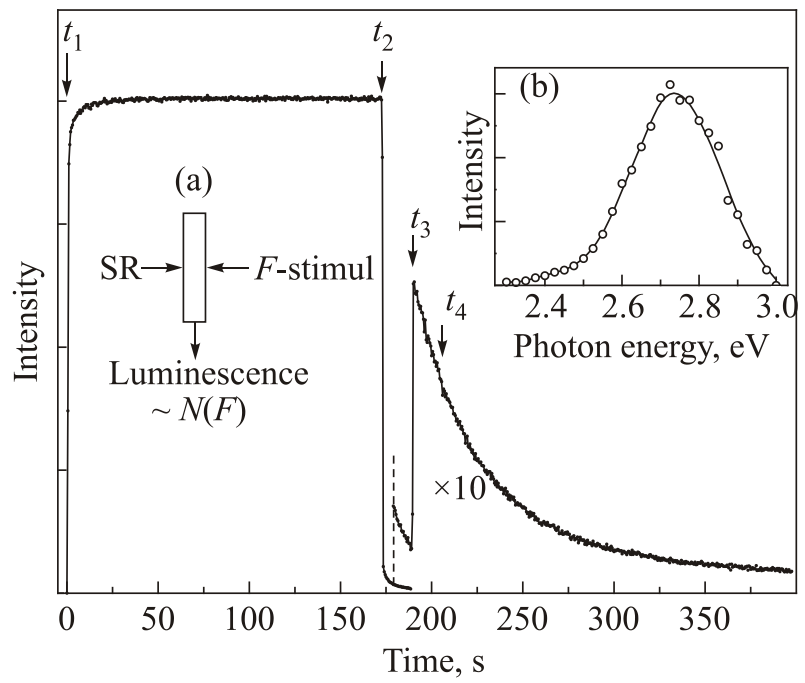

Fig. 1. Time dependence of the intensity of the 3-4 eV luminescence in experiments on the creation of $F$ centres by SR radiation (in the presented case $-38.7 \mathrm{eV}$ photons) at $12 \mathrm{~K}$. The geometry for measuring the creation spectrum by a luminescent method (a). The stimulation spectrum of PSL (measure of $F$ centres) at $80 \mathrm{~K}$ (b).

interval from $t_{1}$ to $t_{2}$ ) perpendicular to the (100) plane by a prescribed number of photons at each of several energies $(7-40 \mathrm{eV})$. The irradiated crystal was afterwards stimulated through a double prism monochromator by the $2.7 \mathrm{eV}$ photons along the $[\overline{1} 00]$ direction in the maximum of the absorption band of radiation-induced $F$ centres [17] (an electron in a field of an anion vacancy). At $12 \mathrm{~K}$, the $2.7 \mathrm{eV}$ photons excite $F$ centres but do not cause their ionization. After the end of $\mathrm{NaCl}$ irradiation and significant decrease of phosphorescence intensity ( $\left.t_{3}\right)$, the time dependence of photostimulated luminescence (PSL) selected through a combination of optical filters $(3-4 \mathrm{eV})$ was recorded. The light sum of this damping PSL (within 15 seconds from $t_{3}$ to $t_{4}$ ) was taken as a measure of $F-H$ pairs formed by SR. Before measuring the next point in the creation spectrum at another energy of exciting photons, the photodestruction of the remaining $F$ centres was performed via more intense $F$ stimulation of the crystal.

The spectra of fast emissions were measured in a wide spectral range of $1.8-7.5 \mathrm{eV}$ at 85 or $295 \mathrm{~K}$ using powerful single electron pulses from the Koval'tchyk-Mesyats type generator (3 ns, the current density 1-180 $\mathrm{A} \mathrm{cm}^{-2}$, $300 \mathrm{keV})$. The luminescence emitted perpendicular to the exciting electron pulses was recorded via a vacuum monochromator or a double prism monochromator. The required corrections for the selectivity of the optical recording channel were performed in the spectra.

\section{Results and discussion}

By comparing the irradiation effects in highly pure $\mathrm{NaCl}$ crystals caused by $\mathrm{x}$ rays, fission fast neutrons or $6-11 \mathrm{eV}$ photons, it was proved long ago that only $e-h$ pairs play the dominant role in the creation of FDs in these 
closely packed crystals [18]. However, these experiments were performed at room temperature, when $E_{F D} \leq E_{g}$ and radiation-induced processes in $\mathrm{NaCl}$ are similar to those in the majority of alkali halides. The opposite inequality, $E_{F D}>E_{g}$ is valid for $\mathrm{NaCl}$ at low temperatures, and the processes of FD creation via $e-h$ recombination (after carriers' vibrational relaxation) or the decay of self-trapping anion excitons are practically frozen [1,2,16,17].

Figure 2 presents the creation spectrum of FDs by SR of various energies $(7-20 \mathrm{eV})$ measured at $12 \mathrm{~K}$ using a luminescent method. The light sum of PSL of $3-4 \mathrm{eV}$ was taken as a measure of radiation-induced $F$ centres (formed as $F-H$ pairs of FDs). According to earlier detailed studies of low-temperature radiation-induced processes in $\mathrm{NaCl}$ and other alkali halides [1,5-7,20,22], the following process is mainly responsible for the appearance of luminescence stimulated in the maximum of the $F$-absorption band of a $\mathrm{NaCl}$ crystal irradiated with a prescribed number of photons with certain energy:

$$
\begin{gathered}
F \ldots H+h v_{\mathrm{stim}}(2.7 \mathrm{eV}) \rightarrow F^{*} \ldots H \rightarrow \alpha \ldots I+h v_{\mathrm{emis}} \\
(3-4 \mathrm{eV}) \rightarrow \mathrm{R}
\end{gathered}
$$

The irradiation creates pairs of neutral FDs $-F-H$ pairs which are stable (we register only such ones) and immobile at low temperature. A further stimulation of the sample with the $2.7 \mathrm{eV}$ photons ( $F$-stimulation) causes the excitation (not ionization) of an $F$ centre up to $2 p$ state $\left(F^{*}\right)$ thus increasing its effective radius and facilitating tunnel transition of an electron from an $F^{*}$ centre to a nearby complementary $H$ centre from the same pair. Such tunnel recharge of $F-H$ into a pair of charged anion FDs $-\alpha-I$ pair (an anion vacancy and a chlorine interstitial ion) was thoroughly studied in many alkali halides (see, e.g., [1,4,6, $24,29]$ ) and is accompanied by the emission in a certain spectral region slightly depending on the interdefect dis-

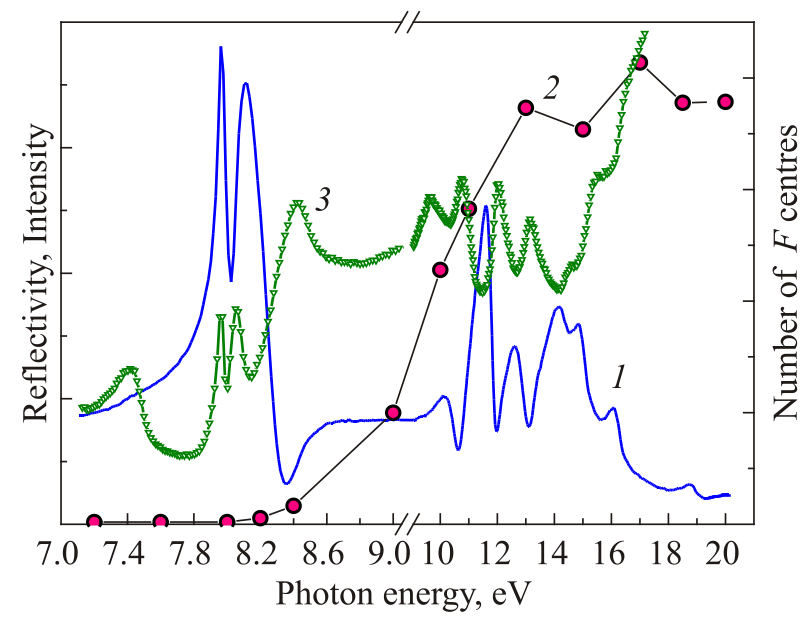

Fig. 2. (Color online) The reflection spectrum (1) and the creation spectrum of $F$ centres (2, see text for details) for a highly pure $\mathrm{NaCl}$ single crystal at $12 \mathrm{~K}$. The excitation spectrum of the $2.67 \mathrm{eV}$ emission of $\mathrm{Br}^{-}$-related centres (3) measured for $\mathrm{NaCl}: \mathrm{Br}(50 \mathrm{ppm})$ at $8 \mathrm{~K}$. tance in a primary pair of FDs. The stimulation spectrum of such PSL arising in our $\mathrm{NaCl}$ sample practically coincides with the shape of the F-absorption band (see Fig. 1(b)). The radiative recharge results in the formation of charged defects ( $\alpha$ and $I$ centres) within a zone of spontaneous recombination and restoration of the regular crystalline structure (R).

It is worth noting that some amount of triplets of spatially correlated defects ( $F, I$ and a self-trapped hole, $V_{K}$ centre) can be formed during photo-irradiation of $\mathrm{NaCl}$. A further $F$ stimulation leads to the appearance of the triplet luminescence of self-trapped excitons (STEs, peaked at $\sim 3.4 \mathrm{eV}$ ) and a similar recovery of a regular lattice. In addition, there are some experimental manifestations that $\mathrm{NaCl}$ is a unique system, in which the energy of a close $F-H$ pair exceeds the level of a relaxed triplet exciton [29], and an $H$ center becoming mobile (for instance, with a rise of temperature) recombines after several jumps with a complementary $F$ centre via the STE triplet state. Nevertheless, in all cases the light sum of PSL is proportional to the number of radiation-induced $F$ centres.

According to Fig. 2, the exciting photons in the region of exciton absorption (7.8-8.4 eV) do not cause the creation of $F$ centres. A weak PSL (possibly, impurity-related) is detected in the spectral region slightly above the band gap $\left(E_{g}=8.9 \mathrm{eV}\right)$, where $s$-conduction electrons are formed. However, a sharp rise of the PSL light sum takes place if the exciting photons of $h v=11-16 \mathrm{eV}$ form hot (nonrelaxed) $p$ - and $d$-conduction electrons [23,28]. Nevertheless, the energy of these exciting photons is insufficient to cause the multiplication of electronic excitations (MEE), when a hot conduction electron forms via Auger-type processes a secondary anion exciton or a secondary $e-h$ pair (see, e.g., [33-35]). The MEE processes in pure and Ag-doped $\mathrm{NaCl}$ crystals were studied in detail [19,22,28]. Figure 2 presents also the excitation spectrum for the $\sim 2.7 \mathrm{eV}$ emission connected with $\mathrm{Br}^{-}$impurity ions measured using SR at $8 \mathrm{~K}$ in a $\mathrm{NaCl}: \mathrm{Br}(50 \mathrm{ppm})$ single crystal. Similar to the case of a triplet STE emission [28], a sharp rise of impurity-related emission efficiency occurs at $h v>15 \mathrm{eV}$ and is caused by the MEE process related to the formation of secondary anion excitons.

So, the enhanced efficiency of stable $F$-centre creation by photons of $12-16 \mathrm{eV}$ can be explained by a nontrivial mechanism of FD creation via hot $e-h$ recombination which is demonstrated at a simplified energy-band diagram of $\mathrm{NaCl}$ (see Fig. 3). The energy released at the recombination of a totally relaxed $e$ with an $h$ that underwent a vibrational relaxation toward the top of a valence band (arrow 2, the so-called cold $e-h$ recombination) or with a self-trapped hole $\left(V_{K}\right.$ centre, arrow 3$)$ is insufficient for the creation of an $F-H$ pair with a relatively large interdefect separation (in other case only a short-lived defect pair can be formed). However, the energy released at the recombination between only partly relaxed $e$ and $h$ (arrow 4 , hot $e-h$ recombination) 


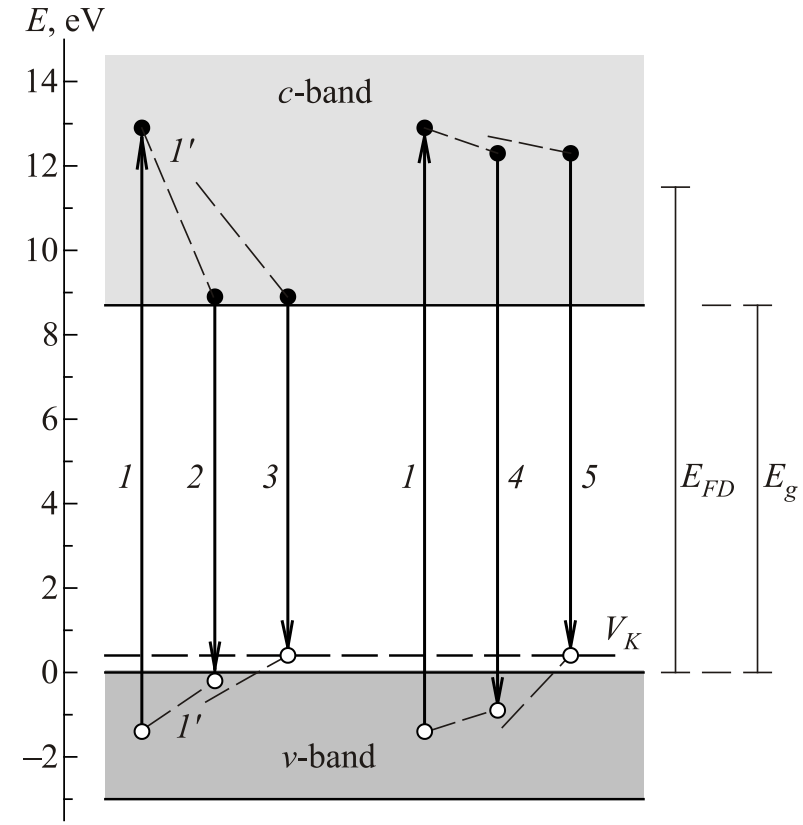

Fig. 3. A simplified energy-band diagram for a $\mathrm{NaCl}$ crystal at low temperature. Arrows 1 demonstrate the absorption of an exciting photon, the inclined dashed arrows show nonradiative (vibrational) relaxation of charge carriers inside conduction (c-band) and valence bands ( $v$-band). Arrows $2-5$ present the energy released via $e-h$ recombination at different stages of carriers' intraband relaxation. The energetic level related to self-trapped holes is marked as $V_{K} ; E_{g}$ and $E_{F D}$ - threshold energy for the creation of an $e-h$ pair or a stable pair of Frenkel defects, respectively.

exceeds the threshold energy for $F-H$ creation, $E_{F D}$. It is generally accepted, that in alkali halides valence holes rapidly undergo self-trapping, forming $V_{K}$ centres totally immobile at low temperatures. So, the recombination of a hot $p$ - or $d$-electron with $V_{K}$ is a more probable version of hot recombination in $\mathrm{NaCl}$. Arrow 5 demonstrates such hot recombination resulting in the formation of an $\mathrm{F}-\mathrm{H}$ pair.

Figure 4 shows the creation spectrum of stable $F$ centres in a spectral region of 25-39 eV where each exciting photon via MEE processes forms in a $\mathrm{NaCl}$ crystal two or even three $e-h$ pairs [28]. The number of $F-H$ pairs (i.e., the PSL light sum) changes only slightly with the rise of the exciting photon energy from 12.5 to $27 \mathrm{eV}$, because only one hot $e-h$, able to create an $F-H$ pair, is produced by each of these photons (the second $e-h$, if formed, is a cold one). A photon of $h v=30-33 \mathrm{eV}$ can form up to three $e-h$ pairs in $\mathrm{NaCl}$ [28]. However, the creation efficiency of $F$ centres just doubles in this spectral region, because only two hot $e-h$ pairs contribute to the creation of stable $F-H$ pairs, while the third $e-h$ is a cold one.

Of particular interest is the creation of $F$ centers by the 33-34 eV photons that form cation excitons [21]. According to Fig. 4, a deep dip of the creation efficiency of $\mathrm{F}-\mathrm{H}$ pairs is observed in the region of cation exciton absorption (see an antibate narrow reflection peak at $33.4 \mathrm{eV}$,

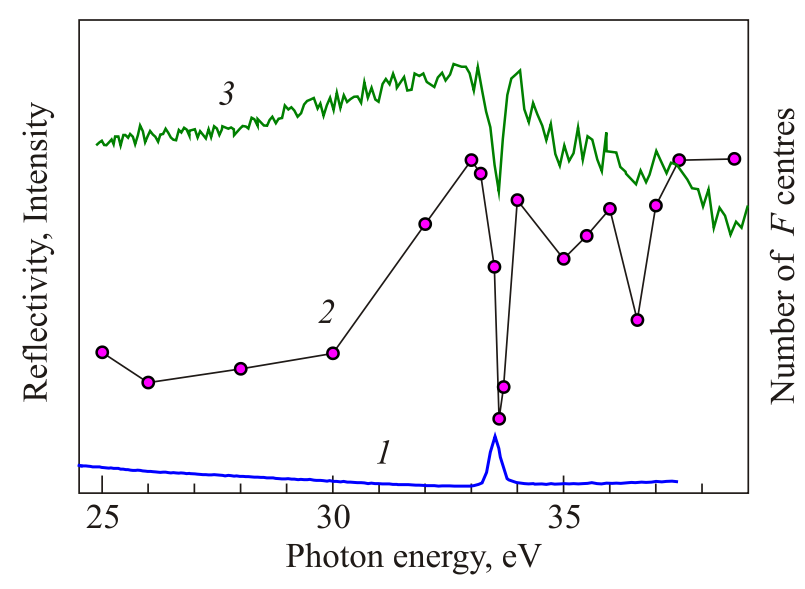

Fig. 4. (Color online) The reflection spectrum (1) and the creation spectrum of $F$ centres (2, see text for details) for a highly pure $\mathrm{NaCl}$ single crystal at $12 \mathrm{~K}$. The excitation spectrum of the $5.17 \mathrm{eV}$ emission of $\mathrm{Ag}^{+}$impurity centres (3) measured for $\mathrm{NaCl}: \mathrm{Ag}(60 \mathrm{ppm})$ at $8 \mathrm{~K}$.

curve 1). This dip remains even if the reflection losses are taken into account. It was shown earlier that a cation exciton in $\mathrm{NaCl}$ decays into an anion exciton and two $e-h$ pairs [28]. This conclusion was based on the analysis of the excitation spectrum for impurity emission in $\mathrm{NaCl}: \mathrm{Ag}$ (600 ppm). The deep dip in the region of $2 p 3 s$ cation exciton creation was ascribed to the formation of an anion exciton during a complex MEE process, while $e-h$ processes dominate in the excitation of $\mathrm{Ag}^{+}$centres at low temperatures. We have detected a similar behavior of the $\mathrm{Ag}^{+}$-emission (pronounced dip at $33.4 \mathrm{eV}$ ) in case of low concentration of silver impurity (Fig. 4, curve 3 ).

The efficiency of the $F$ centre creation at $33.4 \mathrm{eV}$ is approximately the same as at $12.5-27 \mathrm{eV}$ when not more than one hot $e-h$ pair is formed by each exciting photon. The energy excess of about $24 \mathrm{eV}$ remaining after the formation of an anion exciton is enough, due to energetic reasons, to form two $e-h$ pairs, but only one of them would be sufficiently hot for FD creation. So, only a hot $e-h$ pair with energy exceeding $E_{F D}$, formed together with an anion exciton and the second, cold $e-h$ pair at the decay of a cation exciton, is able to create an $F-H$ pair at $12 \mathrm{~K}$.

Under conventional irradiation conditions, a fraction of hot $e-h$ recombination is small, but the recombination probability significantly increases in case of high density excitation. There are experimental manifestations that hot $e-h$ recombination contribute to radiation damage in WGMs with $E_{F D}>E_{g}$ under irradiation with $\sim \mathrm{GeV}$ swift heavy ions providing extremely high EE density along ion tracks (see [12-15] and references therein). In principal, the radiation resistance of these WGMs can be enhanced via purposeful doping due to the solid-state analogue of the Franck-Hertz effect. The latter was revealed in alkali halides doped with $\mathrm{Tl}^{+}$or $\mathrm{Ag}^{+}$ions [35]: a hot conduction electron formed by a photon of $h v>E_{g}$ can lose its energy 
excess not only via vibrational relaxation (arrows $1^{\prime}$ in Fig. 3), but also via the direct excitation of an impurity centre up to the excited state (located within the energy gap), while a subsequent recombination of the cooled carriers provides energy lower than $E_{F D}$, i.e., is insufficient for an FD pair creation. So, the Franck-Hertz effect was considered as some kind of "luminescent protection" against the radiation damage induced by hot $e-h$ recombination $[12,14,15,36,37]$. However, several limitations do not allow to use this approach for significant increase of material radiation resistance [15].

In $\mathrm{NaCl}$ at room temperature, the value of $E_{F D}$ is close to $E_{g}$. Therefore, as it was stated long ago [18], FDs could be created via cold $e-h$ recombination. This fact was confirmed once again by measuring the creation spectrum of $F$ centres by SR in $\mathrm{NaCl}: \mathrm{Tl}$ at $295 \mathrm{~K}$ using a similar luminescent method [29]. At room temperature, $H$ centres are highly mobile and do not exist as single centres, therefore, the light sum of impurity luminescence stimulated in the F-absorption band was taken as a measure of radiation-induced $F$ centres (see, e.g., [33] for details). It was clearly shown that the $F$-centre creation efficiency doubles with the rise of the exciting photon energy from 15 to $18 \mathrm{eV}$, i.e., at the beginning of MEE process at $295 \mathrm{~K}$, when an exciting photon forms two cold $e-h$ pairs each of which is already able to create an $F-H$ pair. At the same time, stable $F$ centres can survive at $295 \mathrm{~K}$ only if complementary $H$ centres become stabilized in the form of trihalide molecules $\mathrm{X}_{3}^{-}$with different microstructure [38]. It was shown earlier that there are favorable conditions for $\mathrm{X}_{3}^{-}$creation, i.e., for the surviving of stable $F$ centres, under MEE conditions when groups of spatially correlated EEs/defects are formed [33].

It has been already mentioned that besides stable FDs the irradiation causes the formation of significantly higher amount of short-lived defect pairs in alkali halides. Creation of short-lived $F-H$ and $\alpha-I$ pairs was detected via corresponding absorption bands under crystal irradiation with nanosecond electron [2,31] or picosecond laser pulses [2,39]. It is worth noting that manifestations of short-lived FDs are also clearly pronounced in the spectra of the so-called intraband luminescence (IBL) discovered in many alkali halides long ago [40,41]. This very fast (subnanosecond) emission is connected with radiative transitions of hot electrons between the levels of the conduction band, i.e., arises via process competitive to nonradiative vibrational relaxation of hot conduction electrons (arrows $1^{\prime}$ in Fig. 3). IBL only slightly depends on temperature and in case of high temperature, when other intrinsic and impurity/defect emissions are usually quenched, has a structureless continuous spectrum. However, the IBL spectra in $\mathrm{RbCl}$ and $\mathrm{KBr}$ contain several minima connected with the radiation-induced absorption bands of short-lived FDs (especially well-pronounced are bands of $F$ and $H$ centres) [42].

Figure 5 presents the spectra of fast emission $(\tau<2 \mathrm{~ns})$ measured for $\mathrm{NaCl}$ at 85 and $295 \mathrm{~K}$ under irradiation with

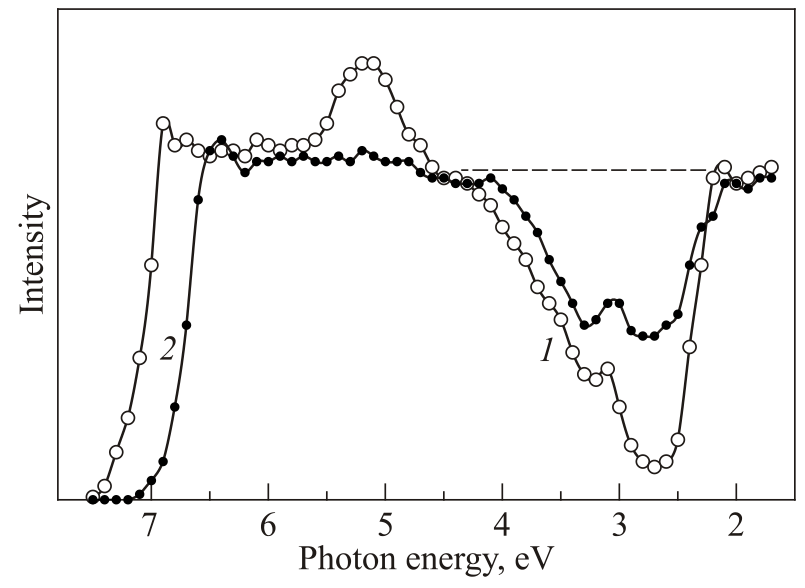

Fig. 5. The spectra of fast ( $\tau<2 \mathrm{~ns}$ ) luminescence for a $\mathrm{NaCl}$ single crystal at $85 \mathrm{~K}(1, \circ)$ and $295 \mathrm{~K}(2, \bullet)$ under irradiation by single $300 \mathrm{keV}$ electron pulses.

single 300-keV electron pulses (duration of $3 \mathrm{~ns}$ ). Again, the continuous IBL spectrum of $\mathrm{NaCl}$ at $295 \mathrm{~K}$ is distorted by rather deep minima in the region of $2.3-4 \mathrm{eV}$. However, in this case the reabsorption structure connected with short-lived FDs has a more complex shape and does not coincide with the bands of $F$ and $H$ centres (peaked at $\sim 2.7$ and $3.8 \mathrm{eV}$, respectively $[1,2,17])$. In our opinion, additional distortion of IBL in $\mathrm{NaCl}$ is tentatively connected with some amount of $H$ centres localized nearby impurity ions (i.e., $H_{A}(\mathrm{Li})$ with absorption at $~ 3.4 \mathrm{eV}$ [24]) or wellpronounced $K$-absorption band at the high-energy wing of $F$ absorption. At $85 \mathrm{~K}$, the spectrum of fast emission also contains the singlet component of STE luminescence ( $\sim 5.3 \mathrm{eV}, \tau \sim 3 \mathrm{~ns}$ ), the thermal quenching of which starts above $50 \mathrm{~K}$ [43]. In contrast to $\mathrm{KBr}, \mathrm{RbBr}$ and $\mathrm{RbCl}$ [42], we did not find the experimental manifestations of the luminescence of one-centre (monohalide) STE at high-energy region of IBL in $\mathrm{NaCl}$ crystals. It should be pointed out that it is extremely difficult to purify $\mathrm{NaCl}$ crystals from calcium impurity ions. A further investigation of the impact of calcium impurity on the creation of FDs in $\mathrm{NaCl}$ still lies ahead.

\section{Conclusions}

The analysis of the creation spectrum of $F$ centres by synchrotron radiation measured at $12 \mathrm{~K}$ using a highly sensitive luminescent method allowed to conclude that, similar to a number of metal oxides [8-11], the recombination of hot (nonrelaxed) conduction electrons and holes causes the creation of pairs of stable anion FDs ( $F-H$ pairs) in a $\mathrm{NaCl}$ crystal with an inequality $E_{F D}>E_{g}$ at cryotemperatures. At low temperatures, neither the decay of anion (7.8-8.4 eV) or cation excitons ( 33.4 eV) nor the recombination of relaxed (cold) electrons and holes lead to the creation of stable Frenkel pairs, the accumulation and aggregation of which determines the radiation resistance of wide-gap materials against prolonged irradiation. 


\section{Acknowledgments}

The research leading to these results has received funding from the Estonian Research Council - Institutional Research Funding IUT02-26.

1. Ch.B. Lushchik and A.Ch. Lushchik, Decay of Electronic Excitations with Defect Formation in Solids, Nauka, Moscow (1989) [in Russian].

2. K.S. Song and R.T. Williams, Self-Trapped Excitons, Springer, Berlin (1996).

3. N. Itoh and A.M. Stoneham, Material Modification by Electronic Excitation, Univ. Press, Cambridge (2000).

4. Ch. Lushchik, J. Kolk, A. Lushchik, N. Lushchik, M. Tajirov, and E. Vasil'chenko, Phys. Status Solidi B 114, 103 (1982).

5. Ch. Lushchik, J. Kolk, A. Lushchik, and N. Lushchik, Phys. Status Solidi A 86, 219 (1984).

6. A.Ch. Lushchik and A.G. Frorip, Phys. Status Solidi B 161, 525 (1990).

7. A. Lushchik, M. Kirm, Ch. Lushchik, and E. Vasil'chenko, Nucl. Instr. Meth. B 166-167, 529 (2000).

8. M. Toulemonde, S. Bouffard, and F. Studer, Nucl. Instr. Meth. B 91, 108 (1994).

9. A.I. Popov and E. Balanzat, Nucl. Instr. Meth. B 166-167, 545 (2000).

10. K. Kimura, S. Sharma, and A.I. Popov, Nucl. Instr. Methods B 191, 48 (2002).

11. N. Itoh, D.M. Duffy, S. Khakshouri, and A.M. Stoneham, J. Phys.: Condens. Matter 21, 474205 (2009).

12. A. Lushchik, Ch. Lushchik, M. Kirm, V. Nagirnyi, F. Savikhin, and E. Vasil'chenko, Nucl. Instr. Meth. B 250, 330 (2006).

13. A. Lushchik, Ch. Lushchik, K. Schwartz, F. Savikhin, E. Shablonin, A. Shugai, and E. Vasil'chenko, Nucl. Instr. Meth. B 277, 40 (2012).

14. A. Lushchik, Ch. Lushchik, V. Nagirnyi, S. Pazylbek, O. Sidletskiy, K. Schwartz, E. Shablonin, A. Shugai, and E. Vasil'chenko, Phys. Status Solidi B 250, 261 (2013).

15. A. Lushchik, Ch. Lushchik, A.I. Popov, K. Schwartz, E. Shablonin, and E. Vasil'chenko, Nucl. Instr. Meth. B 374, 90 (2016).

16. D. Pooley and W.A. Runciman, J. Phys. C 3, 1815 (1970).

17. M. Ikezawa, K. Shirahata, and T. Kojima, Sci. Rep. Res. Tohuku Univ., Ser. 1 52, 45 (1969).

18. Ch.B. Lushchik, G.G. Liidya, M.A. Elango, Sov. Phys. Solid State 6, 1789 (1965) [Fiz. Tverd. Tela 6, 2256 (1964)]. Web of Sci record WOS:A19656189100005.

19. Ch.B. Lushchik, G.K. Vale, E.R. Ilmas, N.S. Rooze, A.A. Elango, and M.A. Elango, Opt. Spectrosc. 21, 377 (1966) [Optika i Spektroskopiya 21, 686 (1965)]. WOS:A19669269100005.
20. E.A. Vasil'chenko, N.E. Lushchik, and Ch.B. Lushchik, Sov. Phys. Solid State 12, 167 (1970) [Fiz. Tverd. Tela 12, 211 (1970)]. WOS:A1970G891100038.

21. S. Nakai, T. Ishii, and T. Sagawa, J. Phys. Soc. Jpn. 30, 428 (1971).

22. J.H. Beamont, A.J. Bourdilon, and M.N. Kabler, J. Phys. C 9, 2961 (1976).

23. J.-F. Himpsel and W. Steinmann, Phys. Rev. B 17, 2537 (1978).

24. A. Lushchik, J. Kolk, N. Lushchik, and A. Frorip, Trudy Inst. Fiz. Akad. Nauk Eston. SSR 58, 25 (1986) [in Russian].

25. V.E. Puchin, A.L. Shluger, and N. Itoh, Phys. Rev. B 47, 10760 (1993).

26. E.A. Kotomin, V.E. Puchin, and P.W.M. Jacobs, Philos. Mag. A 68, 1359 (1993).

27. V. Perebeinos, P.B. Allen, and M. Weinert, Phys. Rev. B 62, 12589 (2000).

28. E. Feldbach, M. Kirm, A. Lushchik, Ch. Lushchik, and I. Martinson, J. Phys.: Condens. Matter 12, 1991 (2000).

29. A. Lushchik, Ch. Lushchik, T. Kärner, V. Nagirnyi, E. Shablonin, and E. Vasil'chenko, Izv. VUZOV, Fizika 52, 95 (2009) [in Russian].

30. K. Tanimura, J. Phys. Soc. Jpn. 65, 3672 (1996).

31. T. Karasawa and M. Hirai, J. Phys. Soc. Jpn. 34, 276 (1973).

32. V. Veligura, G. Hlawacek, R. van Gastel, H.J.W. Zandvliet, and B. Poelsema, J. Phys.: Condens. Matter 26, 165401 (2014).

33. A. Lushchik, I. Kudryavtseva, Ch. Lushchik, E. Vasil'chenko, M. Kirm, and I. Martinson, Phys. Rev. B 52, 10069 (1995).

34. A. Lushchik, E. Feldbach, Ch. Lushchik, M. Kirm, and I. Martinson, Phys. Rev. B 50, 6500 (1994).

35. E. Feldbach, M. Kamada, M. Kirm, A. Lushchik, Ch. Lushchik, and I. Martinson, Phys. Rev. B 56, 13908 (1997).

36. A. Lushchik, Ch. Lushchik, P. Liblik, A. Maaroos, V.N. Makhov, F. Savikhin, and E. Vasil'chenko, J. Lumin. 129, 1894 (2009).

37. A. Lushchik, Ch. Lushchik, T. Kärner, P. Liblik, V. Nagirnyi, E. Shablonin, A. Shugai, and E. Vasil'chenko, Radiat. Meas. 45, 268 (2010).

38. Ch. Lushchik, A. Elango, R. Gindina, L. Pung, A. Lushchik, A. Maaroos, T. Nurakhmetov, and L. Ploom, Semicon. Insulat. 5, 133 (1980).

39. R.T. Williams, B.B. Craig, and W.L. Faust, Phys. Rev. Lett. 52, 1709 (1984).

40. D.I. Vaisburd and I.N. Balychev, JETP Lett. USSR 15, 380 (1972).

41. D.I. Vaisburd and K.E. Evdokimov, Phys. Status Solidi C 2, 216 (2005).

42. Ch.B. Lushchik, A.Ch. Lushchik, E.A. Vasil'chenko, and F.A. Savikhin, Phys. Solid State 37, 284 (1995) [Fiz. Tverd. Tela 37, 525 (1995)].

43. S. Wakita, Y. Suzuki, H. Ohata, S. Tagawa, and M. Hirai, J. Phys. Soc. Jpn. 50, 3378 (1981). 